\title{
Freundlich und konsequent
}

\author{
Annamarie Ryter
}

Ein wichtiges, aber ungeliebtes Instrument der Personalentwicklung ist das Konfliktgespräch. Hinweise für eine gute Vorbereitung und für einen strukturierten Verlauf können Führungskräfte bei dieser heiklen Aufgabe helfen.

Mitarbeitende zu führen macht Spaß. Mit einem motivierten Team lassen sich Berge versetzen. Was aber, wenn es schwierig wird? Wenn Vorgaben der Leitung nicht umgesetzt werden, Bitten ungehört verhallen und der gleiche Mitarbeiter auch nach vier Mahnungen zu spät zur Teamsitzung eintrudelt? Dann ist ein Konfliktgespräch angesagt. Bewährt hat sich dafür folgende Haltung (in Abwandlung vom sogenannten Harvard Prinzip): freundlich im Ton, bereit die Sichtweise des Gegenübers anzuerkennen, aber konsequent in der Sache.

Ein erfolgreiches Konfliktgespräch beginnt bei der seriösen Vorbereitung. Diese dauert nicht selten gleich lang wie das Gespräch selber, beispielsweise 45 Minuten. Wer kein klares Ziel hat, riskiert zur Figur im bekannten Witz zu werden, die am Schluss sagt: »Hauptsache, wir haben darüber geredet. « Ein Konflikt wird so nicht gelöst. Eine Vorbereitung kann entlang von vier Fragen erfolgen:

1.Was genau will ich ansprechen, was stört mich? Wichtig ist dabei, sich genau zu notieren, was vorgefallen ist, also kein Generalvorwurf: »Sie sind unzuverlässig ...«, sondern: »Letzte Woche kamen sie dreimal zu spät und haben zweimal abends das Dienstzimmer nicht aufgeräumt.«

2.Was will ich erreichen? Welches Ziel verfolge ich mit dem Gespräch, was will ich am Ende als Vereinbarung unbedingt haben, wo gebe ich keinesfalls nach, wo bin ich noch offen? Möglich ist, sich schon eine Vereinbarung für den Schluss des Gesprächs zu entwerfen.

3. Was unternehme ich, wenn der Mitarbeiter oder die Mitarbeiterin keine Einsicht zeigt? Packe ich das Problem anders an, hole mir zum Beispiel Unterstützung? Welche Sanktionsmöglichkeiten habe ich überhaupt? Bin ich bereit, Sanktionen (Verweis, Versetzung etc.) auszusprechen?

4.Welchen Rahmen für das Gespräch lege ich fest? Bewährt haben sich ein ungestörter, neutraler Ort und eine Dauer von einer dreiviertel Stunde. Wenn danach keine Lösung gefunden ist, wird ein Folgetermin vereinbart. Bei schwierigen Gesprächen sollte der Mitarbeiter vorher informiert sein, worum es geht, damit er nicht überrumpelt ist. Zwischen Einladung und Gespräch liegen nur ein paar Tage, damit bei einem heiklen Thema sich keine negativen Fantasien verfestigen können.

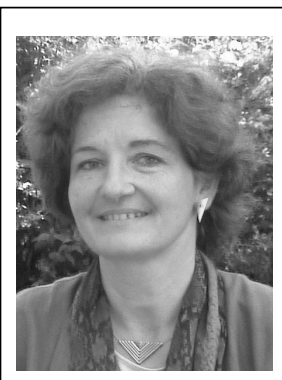

Prof. Dr.phil. Annamarie Ryter ist Hochschullehrerin an der Fachhochschule Nordwestschweiz. Dort bietet sie regelmäßig Weiterbildungskurse zu Führungsthemen, insbesondere zu Mitarbeitergesprächen an. Von Haus aus ist sie Historikerin und Germanistin und besitzt Zertifikate in Systemischer Organisationsentwicklung, Unternehmensberatung und Coaching. Sie hat langjährige Erfahrung in der Beratung und Begleitung von Non-Profit-Organisationen und coacht Führungspersonen. Ryter ist Mitinhaberin des Beratungsunternehmens »bildbar « in Basel. Internet http://www.fhnw.ch (Hochschule für soziale Arbeit, Weiterbildung) Internet http://www.bildbar.ch

Eine Führungsperson hält die Fäden der Gesprächsleitung immer in der Hand. Zugleich versucht sie, zum Gegenüber eine wertschätzende Beziehung beizubehalten. Das folgende Verlaufsschema gibt eine klare Struktur:

- Einstieg: Die Führungsperson gibt Anlass, Rahmen und Dauer des Gesprächs nochmals bekannt. Sie kommt damit rasch zur Sache. Je brisanter das Thema, desto klarer soll es benannt werden. Kein Reden um den heißen Brei - das verunsichert, wirkt zynisch, ist also wenig konstruktiv.

- Eigene Sicht darlegen: Die Führungsperson stellt klar, wo sie die Probleme sieht, was aus ihrer Sicht "Sache" ist. Hier kann sie auf die Vorbereitungen zurückgreifen, auf präzise Beobachtungen, sie vermeidet moralische Vorwürfe, wie etwa »Ich bin enttäuscht von Ihnen ...«

- Sicht des Mitarbeiters, der Mitarbeiterin erfragen: Wie sieht er/sie das Problem?

- Nachfragen und klären, Hintergründe erfassen: Nicht selten verstecken sich hinter äußeren Schwierigkeiten tieferliegende Probleme wie Stress, Überforderung, familiäre Krisen, Teamschwierigkeiten etc. Sie zu kennen, ist für eine optimale Lösung zuweilen unumgänglich.

- Wie weiter? Vorgehen festlegen: Der Blick geht jetzt nach vorne zur erwünschten Veränderung. Die Wunderfrage heißt: »Was brauchen Sie, um meine Forderungen zu erfüllen?« Die Führungsperson gibt also nicht aus lauter Verständnis gleich ihre Ansprüchen auf, sondern überlegt zusammen mit dem Mitarbeiter, wie die Ansprüche erfüllt werden können, welche Mittel es dazu braucht. 


\begin{tabular}{|c|c|c|}
\hline Haltung & Vorgehen & Rahmen \\
\hline \multirow{3}{*}{ 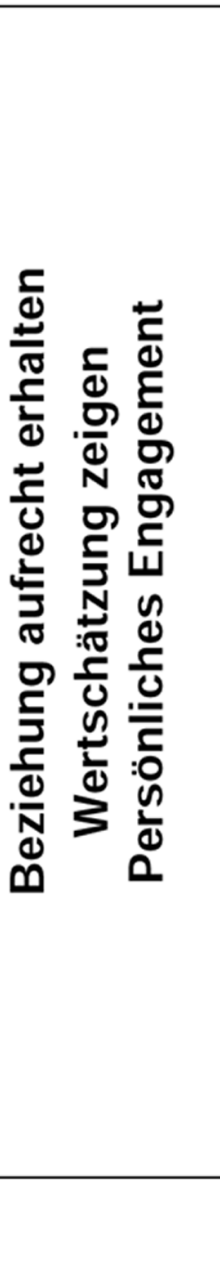 } & $\begin{array}{l}\text { Vorbereitung: } \\
\text { - Was genau will ich ansprechen? } \\
\text { - Was will ich erreichen? } \\
\text { - Was unternehme ich, wenn die Mitarbeiterin } \\
\text { - keine Einsicht zeigt? } \\
\text { - Welchen Rahmen für das Gespräch lege ich } \\
\text { fest? }\end{array}$ & \multirow{2}{*}{ 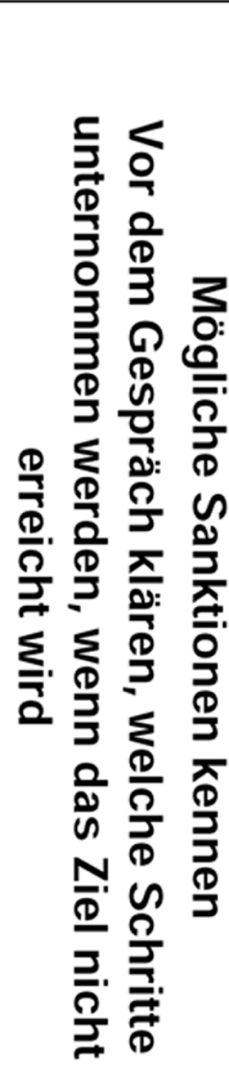 } \\
\hline & \begin{tabular}{|l} 
Durchführung: \\
- Einstieg: Anlass und Rahmen des Gesprächs \\
- deklarieren \\
- Eigene Sicht darlegen, Klartext sprechen \\
- Nachfragen und klären, Hintergründe erfassen \\
- Wie weiter? Vorgehen festlegen \\
- Verbindliche Vereinbarungen treffen \\
- Weiterer Termin zur Überprüfung vereinbaren \\
- Abschluss: eventuell gemeinsame Reflexion \\
des Gesprächs
\end{tabular} & \\
\hline & $\begin{array}{l}\text { Nachbereitung: } \\
\text { - Reflexion, Aktennotiz }\end{array}$ & \\
\hline
\end{tabular}

Abbildung: Die wichtigsten Aspekte von Konfliktgesprächen mit Mitarbeitenden auf einen Blick.

- Verbindliche Vereinbarungen treffen: Hier geht es darum, »Nägel mit Köpfen zu machen «. Was genau soll in Zukunft erreicht werden? Notwendig sind dafür realistische und überprüfbare Ziele. Eine allgemeine Absichtserklärung »Ich werde mich bemühen« reicht nicht.

- Weiterer Termin zur Überprüfung vereinbaren: Unerlässlich ist ein Gespräch nach ein paar Wochen, um die Motivation zu stärken, um Erfolge zu würdigen oder bei Schwierigkeiten die Gelegenheit zu haben, nachzuhaken.

- Abschluss: eventuell gemeinsame Reflexion des Gesprächs: Gerade bei besonders schwierigen Gesprächen ist es sinnvoll, gemeinsam kurz Rückblick zu halten und Eindrücke auszutauschen.

In der Nachbereitung notiert sich die Führungsperson persönliche Eindrücke oder sie erstellt gleich ein offizielles Protokoll, das - auch unterschrieben von der Mitarbeitenden - ins Personaldossier gelegt wird. Diese Leitlinien geben Struktur, garantieren jedoch noch nicht den Erfolg eines schwierigen Gesprächs. Gefragt ist immer eine Balance aus Flexibilität und Hartnäckigkeit der Führungsperson - passend zur Situation und zum Gegenüber. Hier individuell den richtigen Ton zu finden, bleibt auch für Profis eine spannende Herausforderung! •

\section{Literatur:}

Baitsch, Christof/Steiner, Ellen (2004): Zwei tun das Gleiche. Kommunikation zwischen Frauen und Männern im Berufsalltag. (Hg.) Eidgenössisches Justiz- und Polizeidepartement. Zürich vdf Hochschulverlag AG ETH.

15 diskussionswürdige, differenzierte Kurzfilme von je circa fünf Minuten zu heiklen Gesprächen mit Mitarbeitenden, gedreht zum Thema Förderung der Gleichstellung, immer verbunden mit anderen Themen. Die Filme mit Hintergrundmaterial eignen sich sehr gut für Schulungen und Diskussionen im Team.

Schultz von Thun, F./Ruppel, J./Stratmann, R. (2000): Miteinander reden: Kommunikationspsychologie für Führungskräfte. Reinbek bei Hamburg.

Griffige Zusammenfassung der Modelle nach Schulz von Thun spezifisch für Führungskräfte. Eignet sich gut zum Einstieg in die Thematik. 